EGU21-974

https://doi.org/10.5194/egusphere-egu21-974

EGU General Assembly 2021

(c) Author(s) 2021. This work is distributed under

the Creative Commons Attribution 4.0 License.

\title{
Inversion of electromagnetic induction data using a novel wavelet- based and scale-dependent regularization term
}

\author{
Wouter Deleersnyder ${ }^{1,2}$, Benjamin Maveau ${ }^{1}$, David Dudal ${ }^{1,3}$, and Thomas Hermans ${ }^{2}$ \\ ${ }^{1}$ Department of Physics, KU Leuven Campus Kortrijk - KULAK, Kortrijk, Belgium (wouter.deleersnyder@kuleuven.be) \\ ${ }^{2}$ Department of Geology, Ghent University, Gent, Belgium \\ ${ }^{3}$ Department of Physics and Astronomy, Ghent University, Gent, Belgium
}

In frequency domain Electromagnetic Induction (EMI) surveys, an image of the electrical conductivity of the subsurface is obtained non-invasively. The electrical conductivity can be related to important subsurface properties such as the porosity, saturation or water conductivity via Archie's law. The advantage of geophysical EMI surveys is its cost-effectiveness because it is a noncontacting method, one can easily walk with the device or mount in on a vehicle or a helicopter (AEM).

The process of finding the conductivity profile from the collected field data is an ill-posed inverse problem. Regularization improves the stability of the inversion and, based on Occam's razor principle, a smoothing constraint is typically used with a very large number of thin layers. However, the conductivity profiles are not always expected to be smooth. Another alternative is to use a predefined number of layers and to invert for their conductivity and thickness. This can yield sharp contrasts in conductivity. In practice however, the real underground might be either blocky or smooth, or somewhere in between. Those standard constraints are thus not always appropriate.

We develop a new minimum-structure inversion scheme in which we transform the model into the wavelet space and impose a sparsity constraint. This sparsity constrained inversion scheme minimizes an objective function with a least-squares data misfit and a sparsity measure of the model in the wavelet domain. With a solid understanding of wavelet theory, a novel and intuitive model misfit term was developed, allowing for both smooth and blocky models, depending on the chosen wavelet basis. A model in the wavelet domain has both temporal (i.e. low and high frequencies) and spatial resolution, and penalizing small-scale coefficients effectively reduces the complexity of the model.

Comparing the novel scale-dependent wavelet-based regularization scheme with wavelet-based regularization with no scale-dependence, revealed significantly better results (Figure A and B) w.r.t. the true model. Comparing with standard Tikhonov regularization (Figure $C$ and $D$ ) shows that our scheme can recover high amplitude anomalies in combination with globally smooth profiles. Furthermore, the adaptive nature of the inversion method (due to the choice of wavelet) allows for high flexibility because the shape of the wavelet can be exploited to generate multiple 
representations (smooth, blocky or intermediate) of the inverse model.

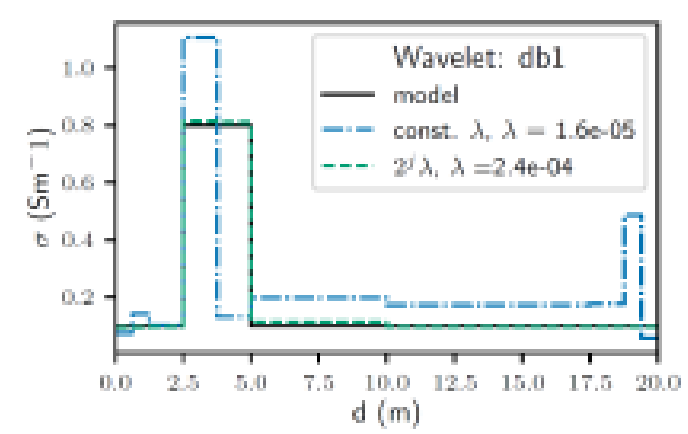

(A)

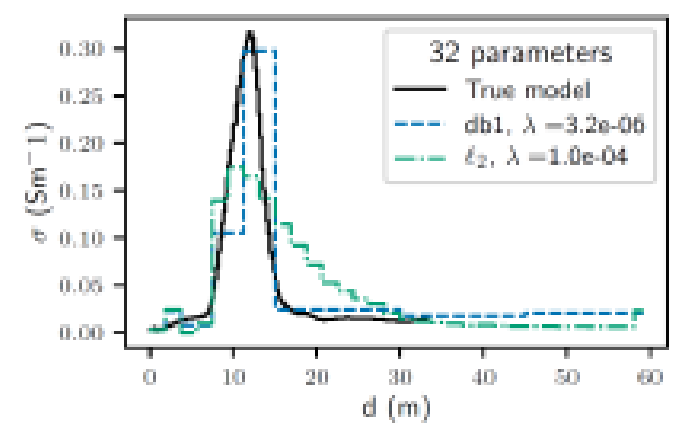

(C)

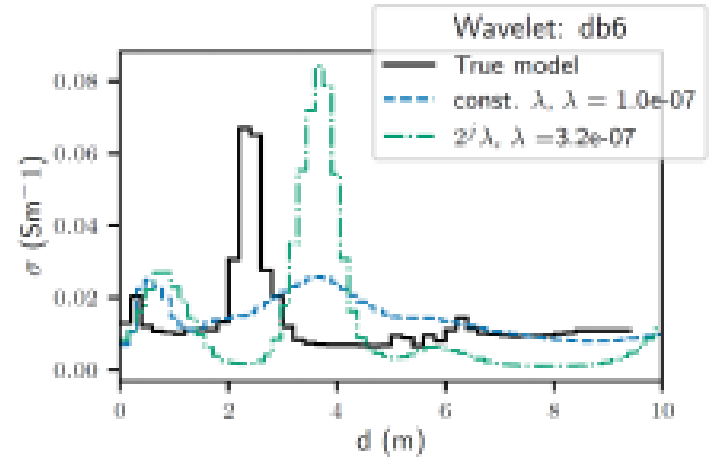

(B)

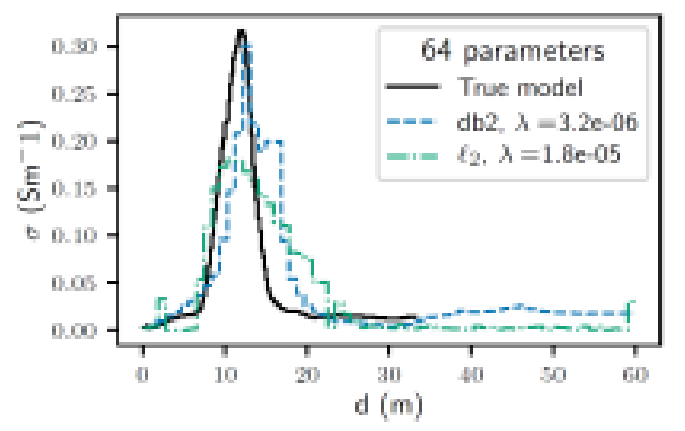

(D)

We have introduced an alternative inversion scheme for EMI surveys that can be extended to any other 1D geophysical method. It involves a new model misfit or regularization term based on the wavelet transform and scale-dependent weighting which can easily be combined with the existing framework of deterministic inversion (gradient-based optimization methods, L-curve criterion for optimal regularization parameter). A challenge remains to select the optimal wavelet, however, the ensemble of inversion results with different wavelets can also be used to qualitatively assess uncertainty. 